\title{
Transvection regulates the sex-biased expression of a fly $X$-linked gene
}

\author{
Authors: Charalampos Chrysovalantis Galouzis ${ }^{1}$, Benjamin Prud'homme ${ }^{1, *}$
}

\begin{abstract}
Affiliations:
${ }^{1}$ Aix-Marseille Université, CNRS, IBDM, Institut de Biologie du Développement de Marseille, Campus de Luminy Case 907, 13288 Marseille Cedex 9, France

*Correspondence to: benjamin.prudhomme@univ-amu.fr
\end{abstract}

\begin{abstract}
Sexual dimorphism in animals results from sex-biased gene expression patterns. These patterns are controlled by genetic sex determination hierarchies that establish the sex of an individual. Here we show that the male-biased wing expression pattern of the Drosophila biarmipes gene yellow, located on the $\mathrm{X}$ chromosome, is independent of the fly sex determination hierarchy. Instead, we find that a regulatory interaction between yellow alleles on homologous chromosomes (or transvection), silences the activity of a yellow enhancer functioning in the wing. This enhancer can be, therefore, active in males (XY) but not in females $(\mathrm{XX})$. This transvection-dependent enhancer silencing requires the yellow intron and the chromatin architecture protein $\operatorname{Mod}(\operatorname{mdg} 4)$. Our results suggest that transvection can contribute more generally to the sex-biased expression of X-linked genes.
\end{abstract}

Main Text: Sexual dimorphism in morphology, physiology and behavior is pervasive in animals. Sexbiased gene expression patterns, deployed during embryonic or adult development, direct the formation of these phenotypic differences between sexes (1). It is well established that the transcriptional regulators of the somatic sex-determination hierarchies directly control sexually dimorphic gene regulation (1-3). However, the different tiers of these hierarchies seem to contribute to this control through a variety of regulatory mechanisms (1-7). To better understand the molecular mechanisms governing sexually dimorphic gene regulation, we examined the dimorphic regulation of the yellow (y) gene in D. biarmipes. This species has evolved a male-specific wing pigmentation spot (Fig. 1A, left panel) $(8,9)$. During late pupal wing development in D. biarmipes males, Yellow spatial distribution prefigures the adult 
pigmentation spot (Fig. 1A, right panel). In contrast, almost no pigmentation pattern appears in adult females (Fig. 1B, left panel), and only a handful of wing cells produce a high level of Yellow, forming a typical dotted, stochastic pattern (Fig. 1B, right panel) $(8,10)$.
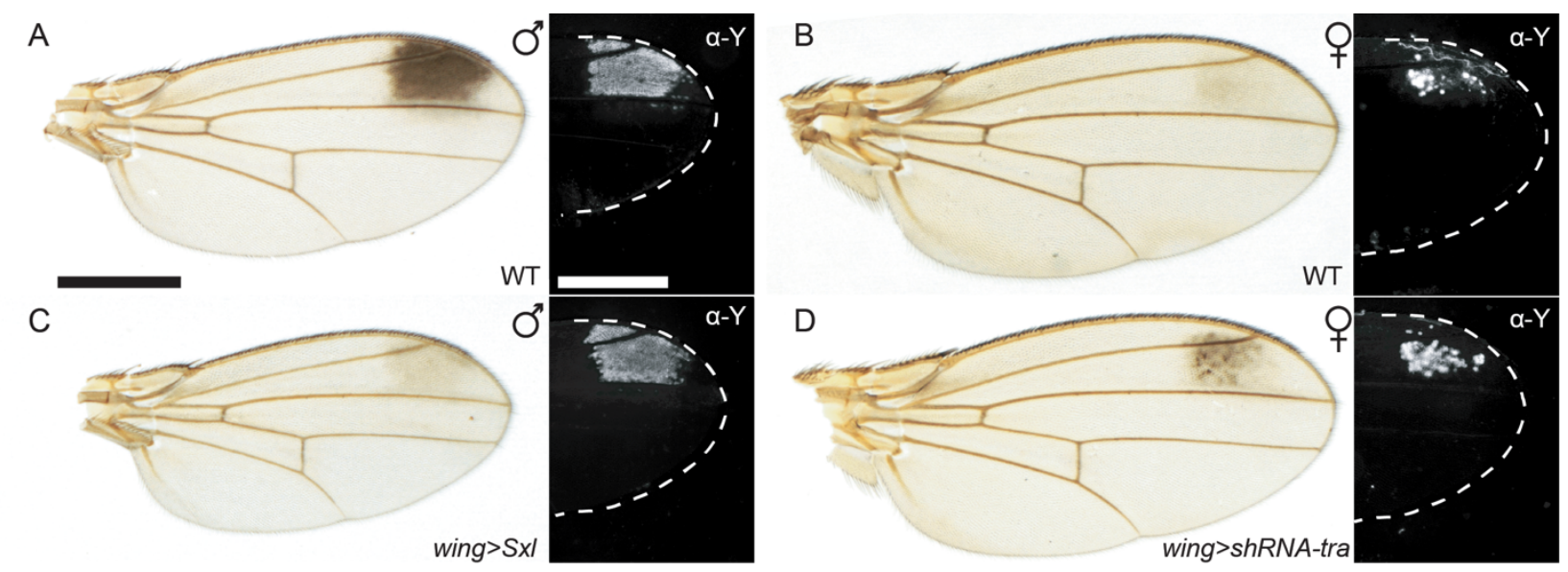

Fig. 1. Yellow sex-biased pattern in the D. biarmipes wing is independent of the sex-determination hierarchy. A male-specific wing pigmentation spot forms in adult D. biarmipes (A, left panel). The wing spot is prefigured during late pupal development by the spotted pattern of Yellow (revealed with an anti-Yellow antibody staining) (A, right panel). In female, the wing pigmentation level is almost uniform, a very faint spot appearing in some individuals (B, left panel), and Yellow is expressed at a high level in just a few cells (B, right panel). Overexpression of $S x l$ in male wings reduces the black spot intensity to levels found in females $(\mathbf{C}$, left panel), without altering Yellow spatial distribution (C, right panel ). Knocking-down tra in female wings leads to increased pigmentation in the spot region (D, left panel), but, Yellow pattern is unaffected (D, right panel). Antibody staining are performed on young adults, just after wing expansion (in all figures). White dashed lines indicate the wing contour. Scale bar, $500 \mu \mathrm{m}$ (in all figures; unless stated otherwise).

We examined the contribution of the top tier of the somatic sex-determination hierarchy to the regulation of $y$ in the D. biarmipes, focusing on Sex-lethal $(S x l)$ and transformer (tra), which initiates and maintains, respectively, female identity in a cell-autonomous manner in Drosophila (11). For this, we feminized the male wing by overexpressing $S x l$, and, conversely, we masculinized the female wing by knocking-down tra expression, using a wing-specific driver (12). In both cases, this altered the wing pigmentation pattern (Fig. 1C, D, left panels, Fig. S1), revealing the conversion of the cells' sexual identity. Surprisingly, however, in both situations, the spatial pattern of Yellow maintained its original sex-specific expression (Fig. 1C, D, right panels). These data confirm that $y$ expression, although necessary to form a wing spot, is not sufficient, and indicate that shifting the sexual identity of the wing affects pigmentation presumably through the modification of genes other than $y$ that are also involved in wing spot formation $(8,12)$. These 
results suggest that the sex-determination hierarchy does not control the sexually dimorphic regulation of $y$ in D. biarmipes wing.

To understand how $y$ is sexually regulated, we sought to identify the cis-regulatory sequences directing its dimorphic expression in the wing. $y$ expression in the D. biarmipes wing is controlled by, at least, a pair of neighboring enhancers, the wing and the spot enhancers (Fig. 2A), which drive $y$ expression throughout the wing at a low level, and in the presumptive wing spot area at a higher level, respectively (9). The spot enhancer drives a sexually monomorphic activity in reporter assays in D. biarmipes (12) (Fig. S2A, B). This result suggests that the spot sequence does not receive female-specific regulatory inputs required for its silencing; another regulatory segment must be involved to control the sexually dimorphic expression of $y$.

A
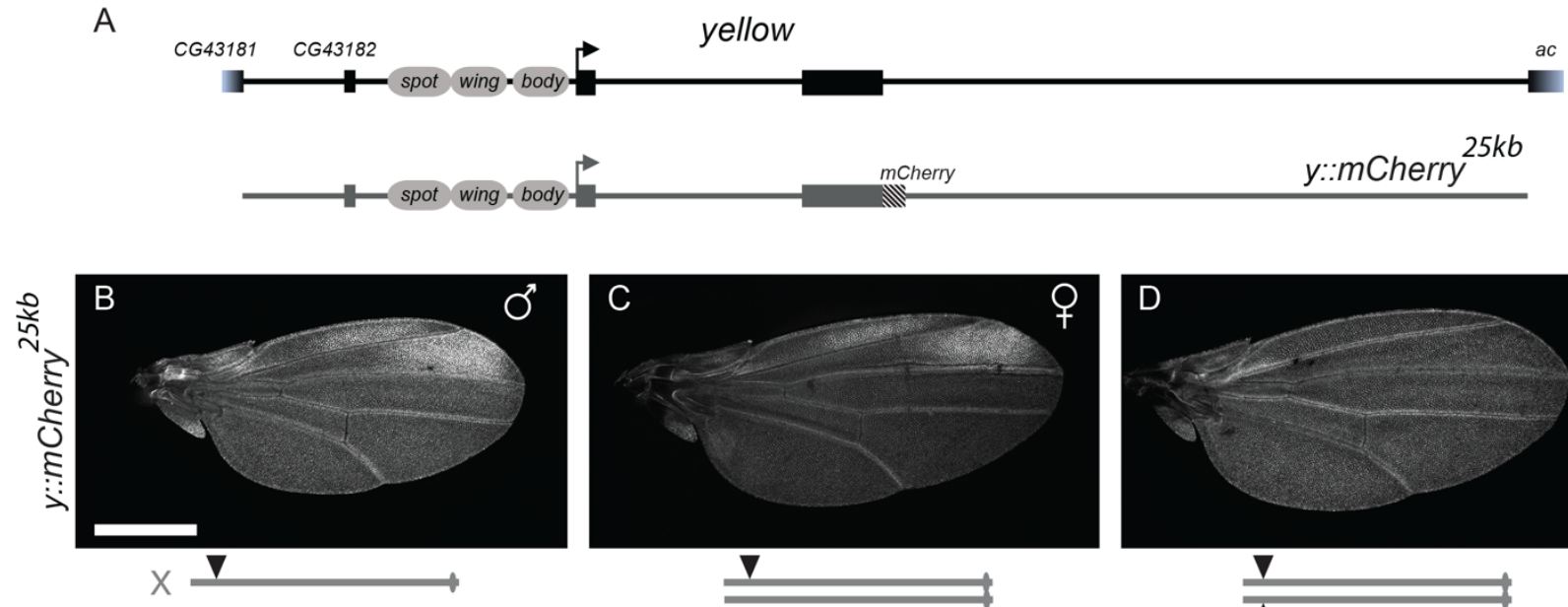

E

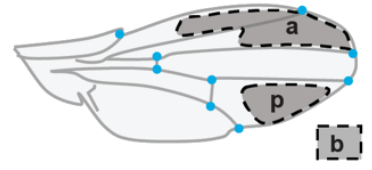

Spotted Expression Index (S.E.I.):

$$
\frac{a \text { m.g.v. }-b \text { m.g.v. }}{p \text { m.g.v. }-b \text { m.g.v. }}
$$
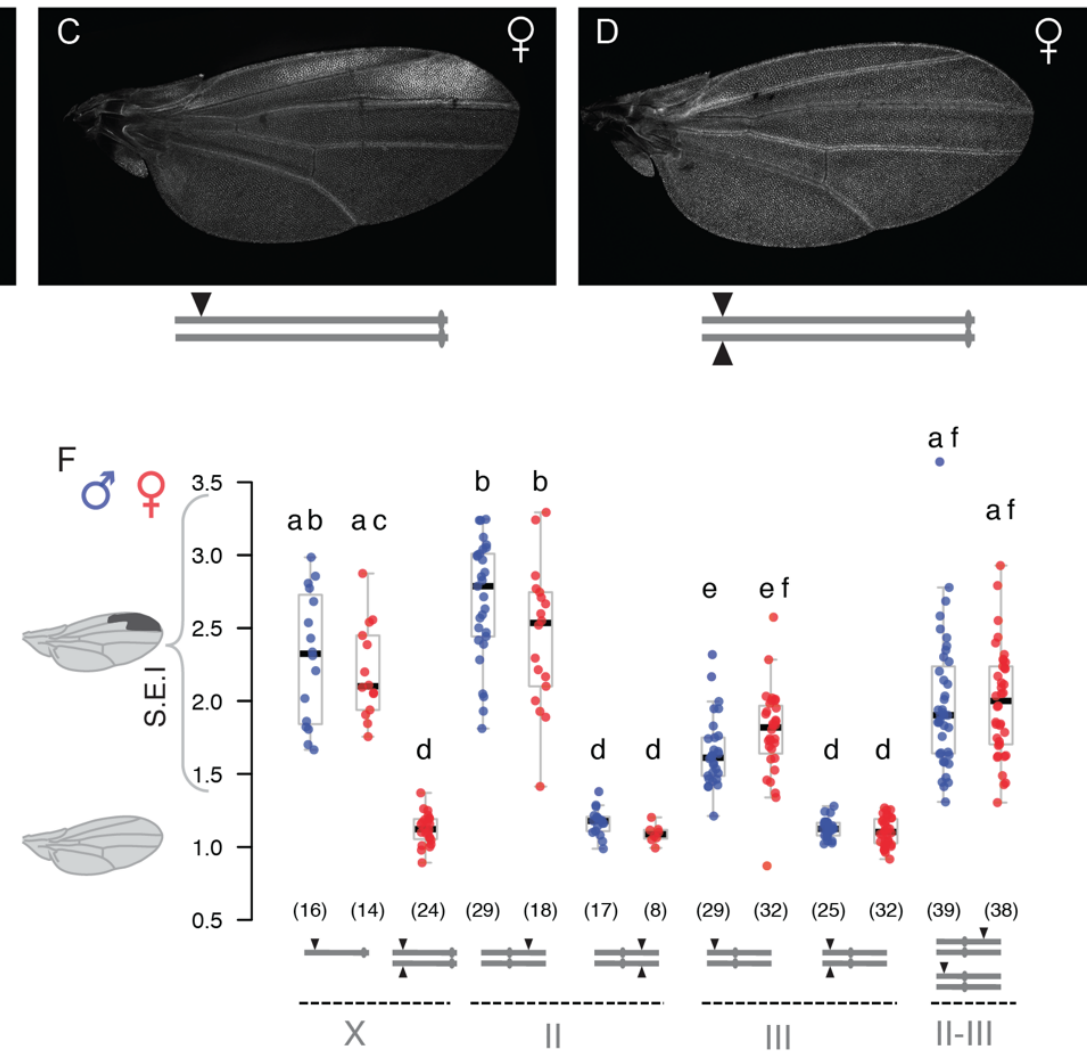

af 
Fig. 2. Sexually dimorphic regulation of $y$ requires functional homolog interaction. Representation of the $D$. biarmipes $y$ locus with the relative positions of the spot, wing and body enhancers, and the 25kb-long fragment cloned from the $y$ locus tagged with $m$ Cherry $\left(y:: m\right.$ Cherry $\left.^{25 k b}\right)$ (A). $y:: m C_{\text {Cherry }}{ }^{25 k b}$ inserted on the X chromosome of $D$. melanogaster drives spotted expression in hemizygous males $(\mathbf{B})$ and heterozygous females $(\mathbf{C})$. In homozygous females, the spotted expression of Yellow::Cherry is silenced (D). In all figures, chromosomes are schematized below each genotype with transgene insertion(s) indicated by black triangle(s). We measured the mean grey value (m.g.v.) in three regions (a, anterior; $p$, posterior; b, background) to calculate the Spotted Expression Index (S.E.I.) (22) (E). Quantification of the S.E.I. of $y:: m C h e r r y{ }^{25 k b}$ insertions on different chromosomes in hemi-, hetero-, homozygous, and trans-heterozygous configurations (F). For all the graphs, each dot represents an individual wing (or abdomen), males are in blue, females in red; numbers in parentheses indicate sample sizes. Also, for comparisons of multiple groups, we used letters (e.g. a, b) to describe the statistical relationship between them and used the following rule for all graphs: groups that share at least one letter (e.g. ab vs. bc) are statistically indistinguishable, and groups that have different letters (e.g. a vs. b) are statistically different ( $\mathrm{p}<0.05$, generalized linear model using a gamma distribution, followed by Tukey's contrasts for multiple comparisons of means).

To define a portion of the $y$ locus that recapitulates its sexually dimorphic expression in the wing, we cloned a 25 kilobase $(\mathrm{kb})$ long fragment, $y^{25 k b}$, encompassing all the coding and non-coding sequences of the gene. We also added a mCherry reporter in frame with the second exon of $y$ to facilitate the detection of the protein product (Fig. 2A). $y$ is X-linked, therefore we inserted the $y:: m C h e r r y^{25 k b}$ reporter construct on the $\mathrm{X}$ chromosome in a $D$. melanogaster $y$ mutant. The $y:: m C h e r r y^{25 k b}$ construct fully rescued the $y$ mutant phenotype in D. melanogaster, although it does not induce the formation of a pigmentation spot, as expected since $y$ is not sufficient to make dark pigment (9), demonstrating its full functionality (Fig. S2C-E). We then examined the distribution of Yellow::mCherry in the wing of freshly emerged adults. In males hemizygous for the transgene, Yellow::mCherry is produced moderately throughout the wing and at a higher level in the spot area, as expected (Fig. 2B). This pattern, hereafter described as a spotted pattern, is similar to the activity of the sum of the wing and spot enhancers in D. melanogaster (9). In females heterozygous for the transgene, $y:: m C_{\text {Cherry }}^{25 k b}$ drove spotted expression (Fig. $2 \mathrm{C}$ ), similarly to hemizygous males. Surprisingly, in D. melanogaster females homozygous for the transgene, Yellow::mCherry is distributed uniformly throughout the wing, but no spotted expression is detected (Fig. 2D).

The different expression patterns driven by the transgene in hemi-/heterozygous and homozygous individuals could result from its insertion on the $\mathrm{X}$ chromosome and the existence of regulatory mechanisms specific to this sex chromosome (13). To explore this possibility, we inserted the $y:: m$ Cherry $^{25 k b}$ construct on different autosomes (chromosomes II or III) and examined its activity in hetero- and homozygous flies, in both sexes. To compare quantitatively the degree of spotted expression 
between genotypes we devised a Spotted-Expression-Index (S.E.I.), which measures the mean intensity of reporter expression in the spot area relative to the mean intensity in a posterior region of the wing (Fig. 2E). Regardless of the sex of the individuals, chromosomal insertions, or vector backbone, $y:: m C h e r r y^{25 k b}$ drove spotted expression in flies heterozygous for the transgene, and uniform wing expression in homozygous individuals (Fig. 2F, Fig. S2F). Together, these results ruled out that the silencing of $y$ spotted expression is controlled by an X chromosome-specific mechanism, or even by female-specific regulators. Instead, they suggest that a regulatory mechanism, common to both sexes, relying on locus copy number difference, controls the spot pattern silencing.

To test whether the absolute copy number influences $y$ regulation, we measured the spotted expression pattern of Yellow::mCherry in trans-heterozygous flies carrying two copies of the transgene inserted on chromosomes II and III, and compared it with the pattern observed in heterozygous or homozygous individuals for each insertion. When the transgenes are present in two copies inserted on different autosomes they drove a spotted expression pattern, in both sexes, similar to either of the heterozygous flies carrying each transgene, but in contrast with the silencing observed in homozygous individuals (Fig. 2F). We thus concluded that the silencing of the $y$ spot pattern is not strictly due to a dose-effect. Instead, the results suggest that the transgenes interact functionally, but only when they are on the same chromosome, to silence the $y$ spot pattern.

To test this idea further, we inserted the $y:: m$ Cherry $^{25 k b}$ transgene at two distinct sites on the $\mathrm{X}$ chromosome, separated by $\sim 5$ megabases $(\mathrm{Mb})$, and measured their combined regulatory activity in the female wing in different configurations. As expected, females heterozygous for each transgene exhibited a spotted pattern, while homozygous females did not (Fig. S2G). Remarkably, when the two copies are at different positions on the same chromosome in trans, or in cis on the same chromatid, the spotted expression is silenced, but not as strongly as in the homozygous flies (Fig. S2G). Furthermore, the genomic sequences flanking the insertion sites are on average closer to one another in the nuclear space when the two transgenes are on the same chromatid, compared to when only one transgene is present (Fig. S2H). These results suggest that functional interaction between the transgenes potentially requires their physical proximity. In the native context, interactions between $y$ alleles is presumably facilitated by the tight pairing of homologous chromosomes, which are aligned end-to-end in all somatic cells in Diptera (14-16). From all these results, we conclude that a regulatory interaction between yellow alleles on homologous chromosomes, a mechanism known as transvection (17-19), controls the sexually dimorphic regulation of 
$y$ in the wing. Since $y$ is X-linked, the transvection-dependent silencing of the spot pattern can only occur in females, which carry two $\mathrm{X}$ chromosomes.

We then wondered if the same mechanism also affects other sex-biased expression patterns of $y$. We examined the male-specific expression of $y$ in the adult posterior abdominal segment (20), which is controlled by the $y$ body enhancer, located nearby the spot and the wing enhancers (Fig. 2A). The sexually dimorphic activity of the body enhancer receives indirect inputs from the sex determination hierarchy (21). We compared the reporter activity of the $y:: m$ Cherry $^{25 k b}$ transgene inserted on the $\mathrm{X}$ chromosome in hemizygous males, hetero- and homozygous females (Fig. S2I-K). As expected, we observed a Yellow::mCherry signal in male posterior segments. By contrast, Yellow::mCherry intensity was low in females, both in hetero- and homozygous flies. This result suggests that the transvection-dependent silencing of $y$ may only affect the spot enhancer.
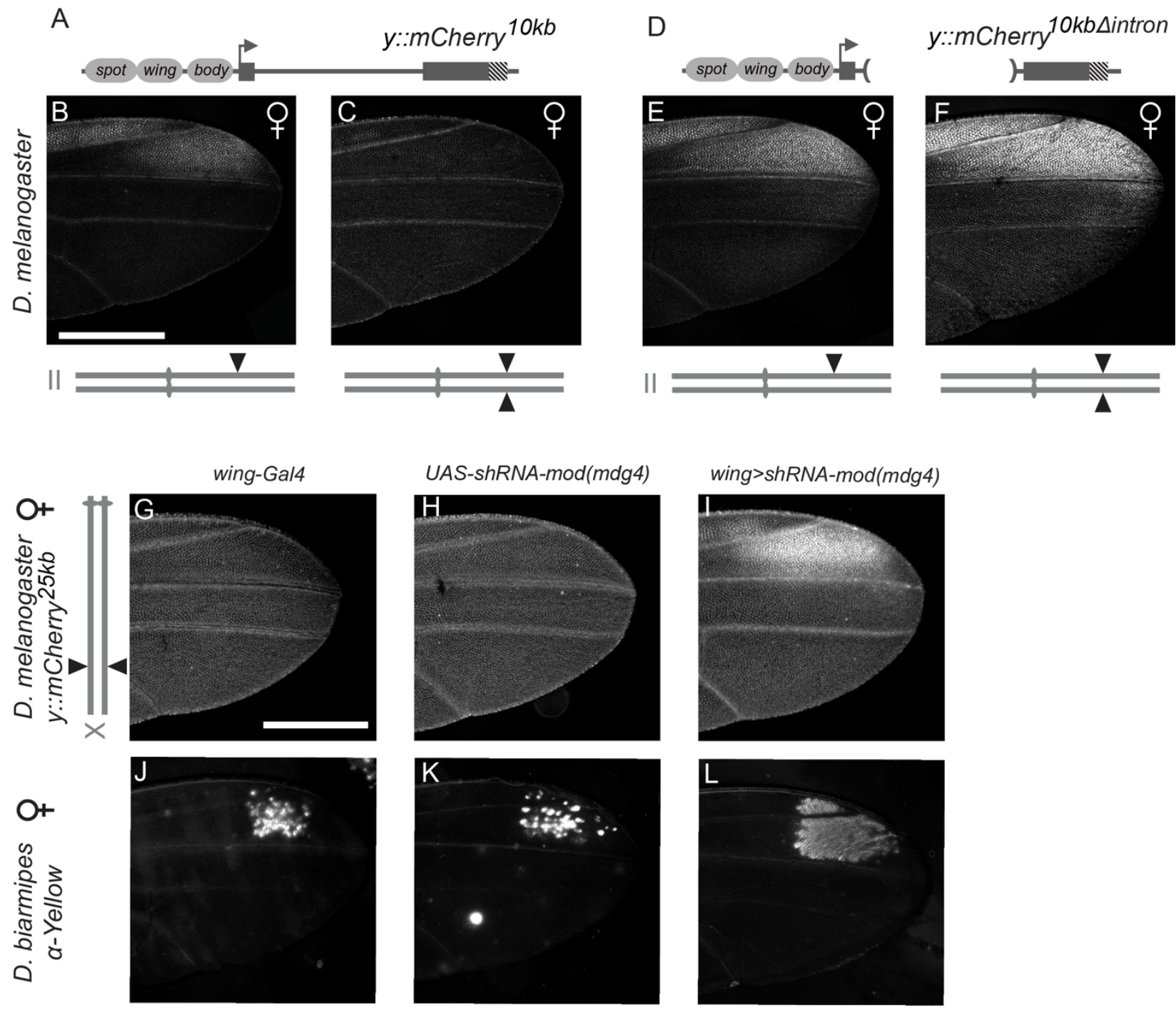
Fig. 3. The $y$ intron and $\bmod (m d g 4)$ are required for the transvection-dependent silencing of the $y$ spot enhancer. $D$. melanogaster females carrying the $y:: m C h e r r y{ }^{10 k b}$ reporter (A) on chromosome II display spotted expression when heterozygous for the transgene (B) and uniform wing expression when homozygous (C). D. melanogaster females carrying $y:: m C_{\text {Cherry }}{ }^{10_{\text {kdaluron }}}$ (D) display spotted expression both when heterozygous (E) or homozygous for the transgene (F). Knocking-down $\bmod (m d g 4)$ in D. melanogaster females abolishes silencing of $y:: m C h e r r y y^{25 k b}(\mathbf{G}-\mathbf{I})$. Similarly, when knocking-down $\bmod (\operatorname{mdg} 4)$ in D. biarmipes female wings, the native Yellow pattern $(\mathbf{J}, \mathbf{K})$ becomes male-like (L).

To localize the sequences involved in the sexually dimorphic regulation of $y$ in the wing we first reduced the $y^{25 k b}$ region to a $y^{10 k b}$ fragment (Fig. S3A) (22), and added $m$ Cherry in frame with the second exon of $y$ (Fig. 3A). The $y:: m$ Cherry $^{10 k b}$ construct behaved like the $y:: m$ Cherry ${ }^{25 k b}$ both in hetero- and homozygous flies (Fig. 3B, C, Fig. S4A). We observed similar results with a construct containing only the $y 5^{\prime}$ and intron sequences of the $y^{10 k b}$ (Fig. S4B-F). This result indicates that the $y$ transcript or exonic sequences are dispensable for the transvection-dependent silencing of the spot pattern.

We then deleted the intron from $y:: m C_{\text {Cherry }}^{10 k b}$ (Fig. 3D). While $y:: m C_{\text {Cherry }}{ }^{\text {rokbantron }}$ and $y:: m C h e r r y^{10 k b}$ drive a similar expression pattern when present as a single copy (Fig. 3B, E, Fig. S4A), we found that the spot

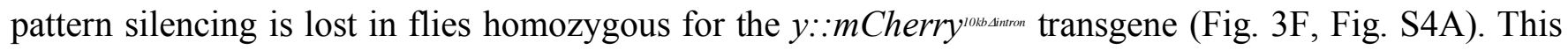
reveals that the $y$ intron is necessary for the transvection-dependent silencing of the spot pattern.

Having identified that transvection shapes the sexually dimorphic regulation of $y$, we ran a genetic screen in D. melanogaster using available alleles or RNAi lines for genes that have been previously associated with this phenomenon. We examined in particular members of the Polycomb group that have been recurrently involved in this mode of gene silencing (23) (Table S2). The only candidate gene that upon knock-down with two independent RNAi lines affected the $y:: m$ Cherry ${ }^{25 k b}$ sex-biased expression was $\bmod (m d g 4)$ (Fig. 3G-I, Fig. S4G). We confirmed this result by knocking down mod(mdg4) in D. biarmipes female wing, which resulted in a male-like Yellow pattern (Fig. 3J-L). These results suggest that $\bmod (m d g 4)$ is required for the transvection-dependent silencing of the $y$ spot activity. Since $\operatorname{Mod}(\operatorname{mdg} 4)$ is involved in chromatin architecture and enhancer blocking (24-26), we speculate that it mediates the interactions between homologous $y$ alleles, possibly by bridging genomic regions to bring the spot enhancers and the introns together.

Altogether, these results identify a mechanism for the regulation of the sex-biased expression of the Xlinked gene $y$ in the wing that is independent of the somatic sex-determination hierarchy, which, in contrast, controls the sex-biased $y$ expression in the body (Fig. S2I-L) (21), and relies instead on transvection (Fig. 
4). Transvection has been documented in different groups of organisms, however, in most cases, interactions are only revealed in mutant contexts or with transgenic constructs $(17,27-29)$. Even in Drosophila melanogaster, where transvection has been studied most extensively, the physiological relevance of this mechanism to gene regulation in a wild type context has long remained questionable (30). Our results suggest that one significant contribution of transvection might be to shape the sexually dimorphic regulation of $\mathrm{X}$-linked genes.
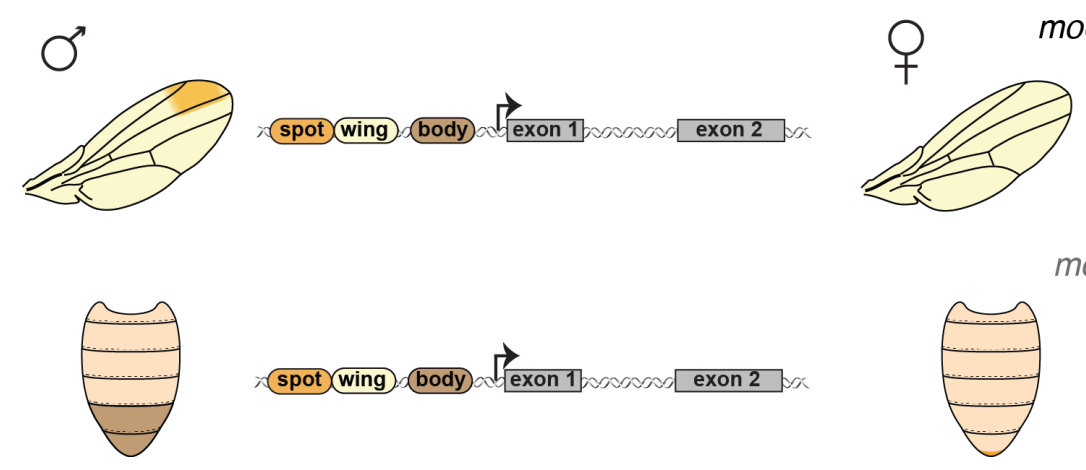

$\bmod (\operatorname{mdg} 4) \quad S x l$, tra

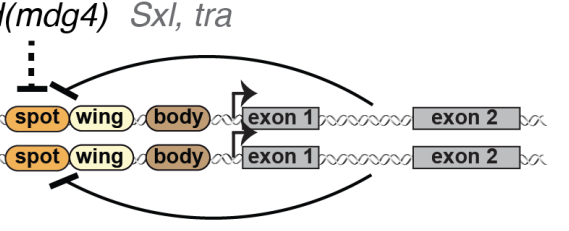

$\bmod (\operatorname{mdg} 4) \operatorname{Sxl}$, tra
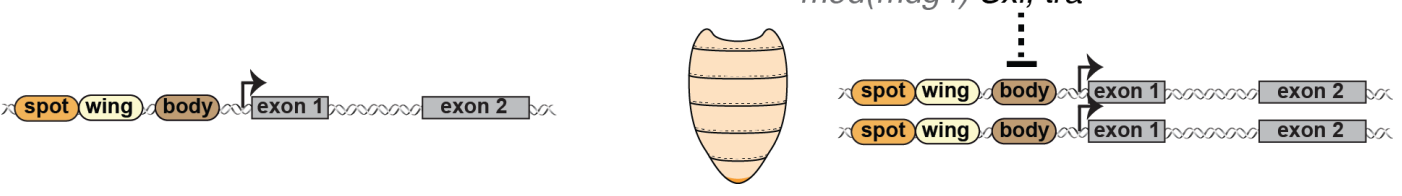

Fig. 4. Regulatory model of $y$ sexually dimorphic expression in the wing and posterior abdomen of D. biarmipes. In males, where $y$ is present in one copy since it is X-linked, the spot and the body enhancers are active (left). By contrast, in females, these enhancers are silenced by two distinct mechanisms: the body enhancer receives repressive inputs from targets of the sex determination hierarchy genes $S x l$ and tra, while the spot is silenced through transvection and uses the non-sex-specific $\operatorname{Mod}(\operatorname{mdg} 4)$ architectural protein (right). 


\section{References and Notes:}

1. T. M. Williams, S. B. Carroll, Nat. Rev. Genet. 10, 797-804 (2009).

2. A. Prakash, A. Monteiro, Curr. Opin. insect Sci. 17, 40-48 (2016).

3. N. Camara, C. Whitworth, M. Van Doren, Curr. Top. Dev. Biol. 83, 65-107 (2008).

4. J. L. Rinn, M. Snyder, Trends Genet. 21, 298-305 (2005).

5. A. Sawala, A. P. Gould, PLoS Biol. 15, e2002252 (2017).

6. X. Sun et al., G3 Genes, Genomes, Genet. 5, 2865-2874 (2015).

7. B. Hudry, S. Khadayate, I. Miguel-Aliaga, Nature. 530, 344-8 (2016).

8. $\quad$ P. J. Wittkopp, J. R. True, S. B. Carroll, Development. 129, 1849-58 (2002).

9. N. Gompel, B. Prud'homme, P. J. Wittkopp, V. A. Kassner, S. B. Carroll, Nature. 433, 481-7 (2005).

10. A. Kopp, J. R. True, Evol. Dev. 4, 278-91 (2002).

11. H. K. Salz, J. W. Erickson, Fly (Austin). 4, 60-70 (2010).

12. L. Arnoult et al., Science. 339, 1423-6 (2013).

13. J. C. Lucchesi, M. I. Kuroda, Cold Spring Harb. Perspect. Biol. 7, 1-21 (2015).

14. N. M. Stevens, J. Exp. Zool. 5, 359-374 (1908).

15. J. AlHaj Abed et al., Nat. Commun. 10, 1-14 (2019).

16. K. Viets et al., Dev. Cell. 51, 341-356.e7 (2019).

17. E. B. Lewis, Am. Nat. 88, 225-239 (1954).

18. J. A. Kennison, J. W. Southworth, Adv. Genet. 46, 399-420 (2002).

19. I. W. Duncan, Annu. Rev. Genet. 36, 521-56 (2002).

20. S. Jeong, A. Rokas, S. B. Carroll, Cell. 125, 1387-99 (2006).

21. M. J. Roeske, E. M. Camino, S. Grover, M. Rebeiz, T. M. Williams, Elife. 7, 1-28 (2018).

22. Materials and methods are available as supplementary material

23. Y. B. Schwartz, V. Pirrotta, Nat. Rev. Genet. 8, 9-22 (2007).

24. O. Kyrchanova, P. Georgiev, FEBS Lett. 588, 8-14 (2014).

25. L. Melnikova et al., Proc. Natl. Acad. Sci. U. S. A. 101, 14806-11 (2004).

26. M. Savitsky, M. Kim, O. Kravchuk, Y. B. Schwartz, Genetics. 202, 601-617 (2016). 
27. M. S. Hogan, D.-E. Parfitt, C. J. Zepeda-Mendoza, M. M. Shen, D. L. Spector, Cell Stem Cell. 16, 275-88 (2015).

28. D. Zhang, L. Bai, Proc. Natl. Acad. Sci. U. S. A. 113, 4428-33 (2016).

29. D. J. Mellert, J. W. Truman, Genetics. 191, 1129-1141 (2012).

30. M. S. Apte, V. H. Meller, Genet. Res. Int. 2012, 430587 (2012).

31. $10.6084 / \mathrm{m} 9$.figshare. 13239110

32. B. Haley, D. Hendrix, V. Trang, M. Levine, Dev. Biol. 321, 482-90 (2008).

33. A. Bassett, J.-L. Liu, Methods. 69, 128-36 (2014).

34. H. Hinaux et al., Dev. Biol. 438, 111-123 (2018).

35. M. Spitzer, J. Wildenhain, J. Rappsilber, M. Tyers, Nat. Methods. 11, 121-2 (2014).

Acknowledgments: We are grateful to the Bloomington Drosophila Stock Center for fly stocks; Flybase for information support; N. Gompel for the anti-Yellow antibody; A. Sultanova for assistance in the collection of abdomens; M. Paris, N. Gompel, J. Ewbank and all the lab members for helpful comments on the manuscript. Funding: This work was funded by the European Research Council under the European Union's Seventh Framework Programme (FP/2007-2013) / ERC Grant Agreement n 615789 (BP). CCG acknowledges funding from the Fondation pour la Recherche Médicale (FRM FDT201904008114). Author contributions: BP conceived the project, BP and CCG designed the experiments, CCG performed all the experiments and the statistical analyses. CCG and BP analyzed the results and wrote the manuscript. Competing interests: Authors declare no competing interest. Data and materials availability: The authors declare that the data supporting the findings of this study are available in the manuscript or supplementary material, and raw image data are deposited in the figshare digital repository (31).

Supplementary Material

Material and Methods

Supplementary Text

Figs. S1 to S2

Tables S1 \& S2 

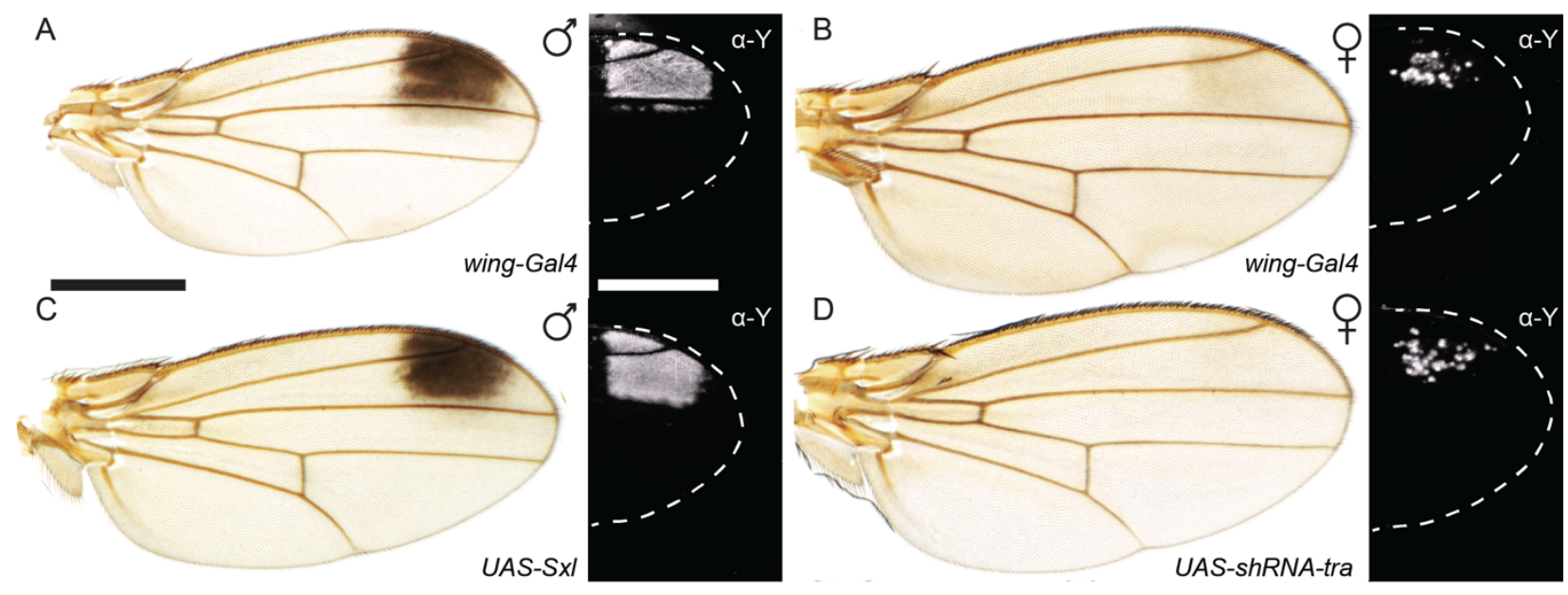

Fig. S1. Yellow sex-biased pattern in D. biarmipes wing is independent of the sex-determination hierarchy. Adult pigmentation and Yellow patterns in the wings of D. biarmipes male wing-Gal4 (A, B), female wing-Gal4 (C, D), male $U A S$-Sxl $(\mathbf{E}, \mathbf{F})$, female $U A S$-shRNA-tra $(\mathbf{G}, \mathbf{H})$. 

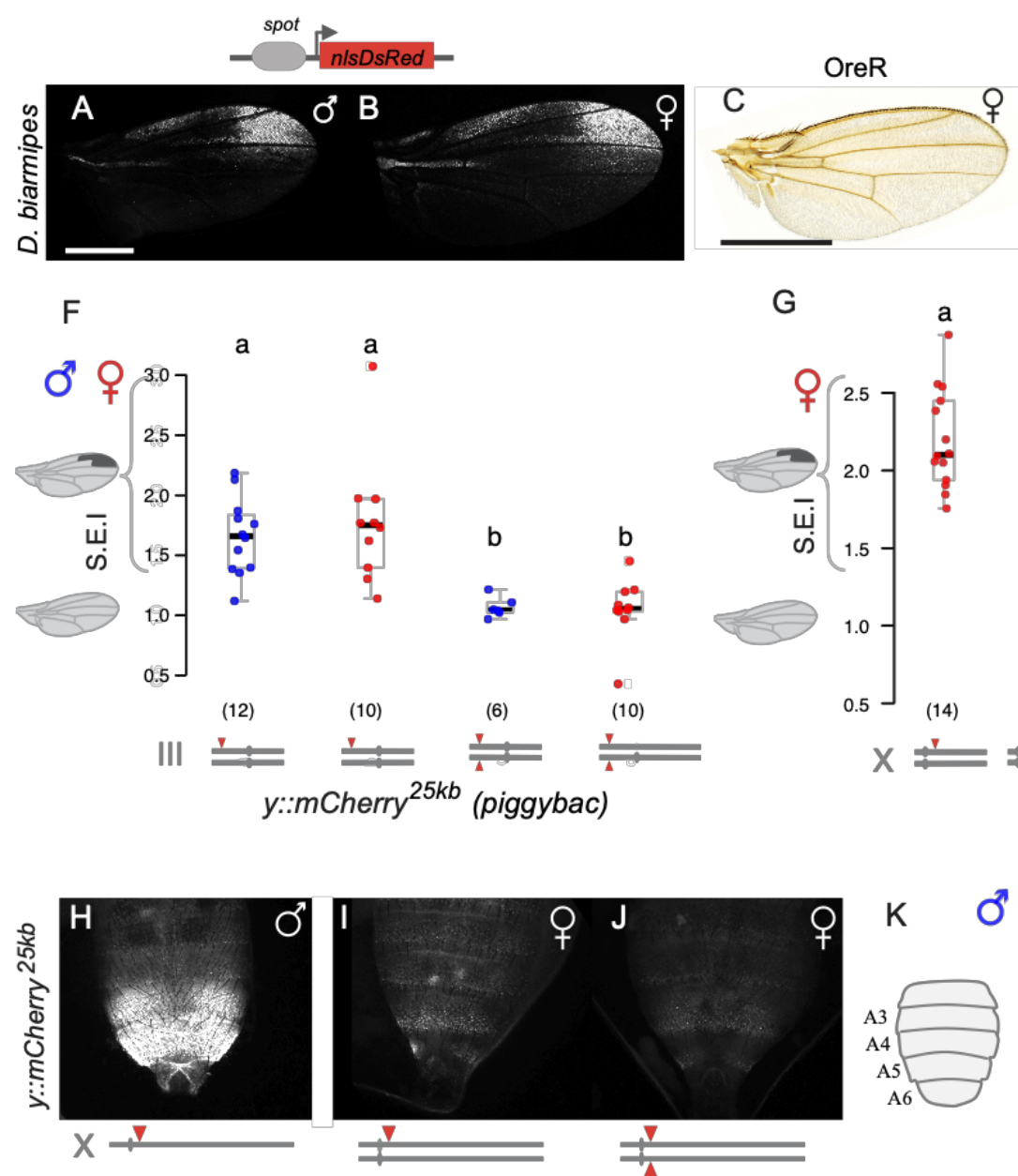
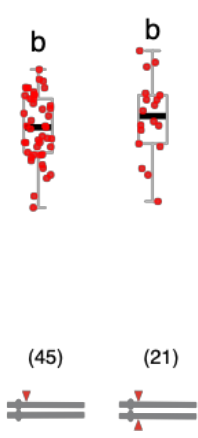

Fig. S2. The spot enhancer drives similar reporter (nlsdsRed) activity in the wing of $D$. biarmipes males (A) and females (B) carrying a transcriptional reporter. Adult wings of D. melanogaster OregonR (C), attPX1 (D) and attPX1 carrying $y:: m C_{\text {Cherry }}{ }^{25 k b}$, which rescues the $y$ mutant pigmentation phenotype (E). The $y:: m C h e r r y{ }^{25 k b}$ construct cloned in a piggyBac vector backbone inserted on $D$. melanogaster chromosome III displays spotted expression in heterozygous males and females as indicated by the S.E.I.. In homozygous males and females, the spotted expression of $y$ is silenced (F). $y:: m C h e r r y{ }^{25 k b}$ inserted at two distinct positions on the $\mathrm{X}$ chromosome of D. melanogaster (5Mb apart) in the different configurations depicted by the schematics. When in trans-heterozygous configuration between the two positions, either on the same or different chromatids, the spotted expression of Yellow::mCherry is significantly decreased revealing the functional interaction between the transgenes at this distance $(\mathbf{G})$. The data points for the attPX1 insertion (first 2 columns on the left) are the same as in Figure $2 \mathrm{f}$. $y:: m C_{\text {Cherry }}^{25 k b}$ inserted on the X chromosome of $D$. melanogaster drives sexually-dimorphic expression in the posterior abdomen(H-J). Quantification of Yellow::mCherry, according to the schematic representation next to the y-axis, shows no significant difference between heterozygous and homozygous females $(\mathbf{K})$. 

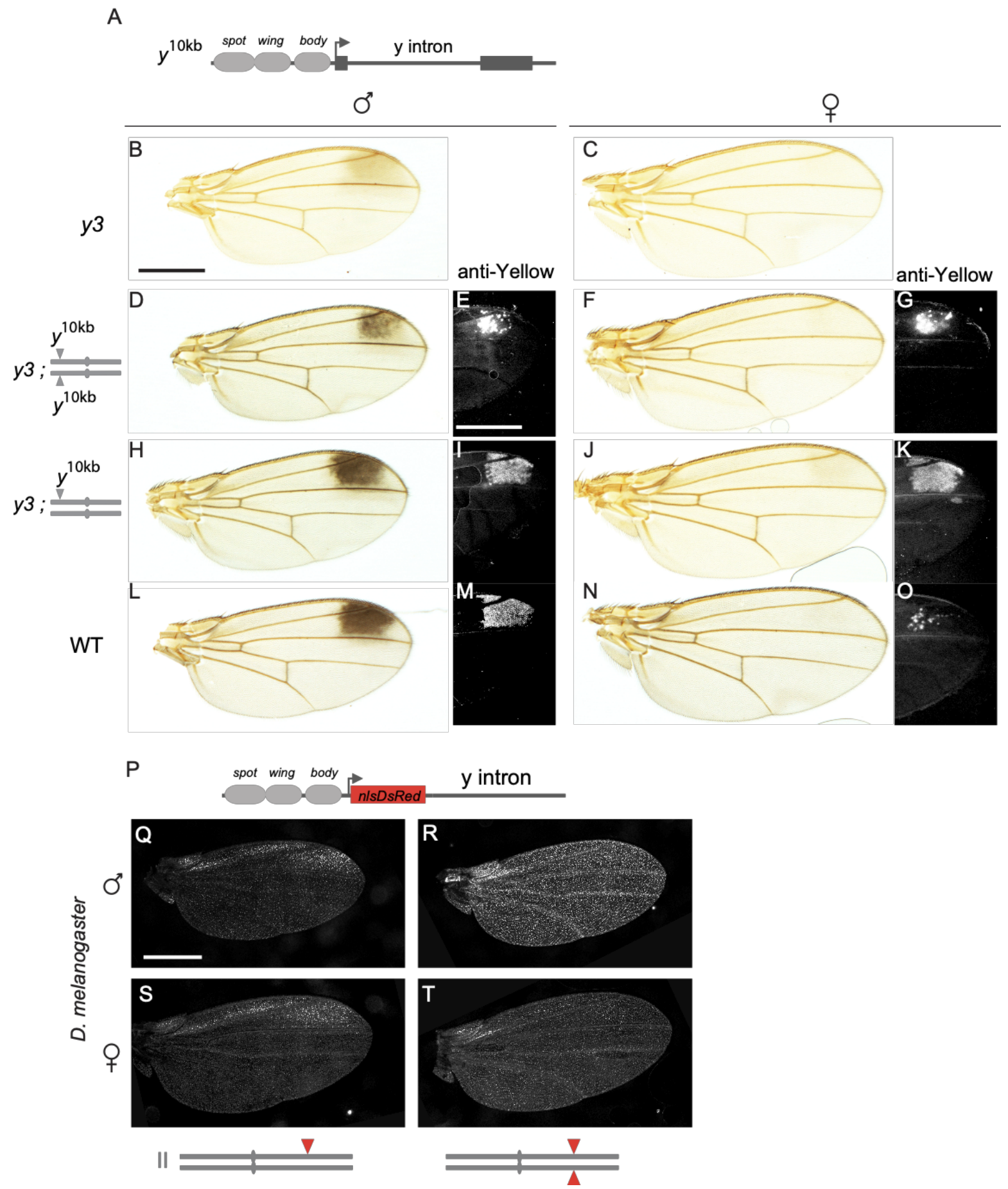

Fig. S3. Schematic representation of the $y^{10 k b}$ construct (A). Adult wings of $y$ mutant $(y 3)$ D. biarmipes male (B) and female $(\mathbf{C})$. Adult pigmentation of D. biarmipes y3 mutants carrying the $y^{10 k b}$ transgene in homozygous configuration in males (D) and females $(\mathbf{F})$ and heterozygous configuration in males (H) and females (J) with the associated Yellow pattern (revealed by an anti-Yellow staining), respectively (E, G, I, 
K). Adult wings and Yellow patterns of wild-type males and females are presented for comparison (L-O). Schematic representation of the $y^{l 0 k b}-n l s D$ sRed transcriptional reporter in which the nlsDsRed is inserted upstream of the $y$ intron $(\mathbf{P})$. This reporter constructs behaves in D. melanogaster like the $y:: m C h e r r y{ }^{10 k b}$ construct, it drives spotted expression when heterozygous $(\mathbf{Q}, \mathbf{S})$, and uniform expression when homozygous $(\mathbf{R}, \mathbf{T})$, in both sexes.

\begin{tabular}{|c|c|c|c|}
\hline BDSC \# & Gene & Flybase ID & spot silencing \\
\hline RNAi lines & & & \\
\hline 29734 & BEAF-32 & |FBst0029734 & Negative \\
\hline 34069 & Caf1-55 & FBst0034069 & Negative \\
\hline 33903 & Cp190 & FBst0033903 & Negative \\
\hline 42536 & Cp190 & FBst0042536 & Negative \\
\hline 40850 & CTCF & FBst0040850 & Negative \\
\hline 31941 & Dref & FBst0031941 & Negative \\
\hline 27993 & $\mathrm{E}(\mathrm{z})$ & FBst0027993 & Negative \\
\hline 33659 & $\mathrm{E}(\mathrm{z})$ & FBst0033659 & Negative \\
\hline 61903 & Elba2 & FBst0061903 & Negative \\
\hline 61180 & HIPP1 & FBst0061180 & Negative \\
\hline 32995 & $\bmod (\operatorname{mdg} 4)$ & FBst0032995 & Positive \\
\hline 33907 & $\bmod (\operatorname{mdg} 4)$ & FBst0033907 & Positive \\
\hline 28343 & $\mathrm{pbl}$ & FBst0028343 & Negative \\
\hline 33945 & Pcl & FBst0033945 & Negative \\
\hline 33946 & $\mathrm{Pcl}$ & FBst0033946 & Negative \\
\hline 42926 & pho & FBst0042926 & Negative \\
\hline 35297 & Psc & FBst0035297 & Negative \\
\hline 31612 & Sce & FBst0031612 & Negative \\
\hline 33704 & Set1 & FBst0033704 & Negative \\
\hline 32473 & Sfmbt & FBst0032473 & Negative \\
\hline 33906 & $\mathrm{Su}(\mathrm{Hw})$ & FBst0033906 & Negative \\
\hline 34006 & $\mathrm{Su}(\mathrm{Hw})$ & FBst0034006 & Negative \\
\hline 31191 & $\mathrm{Su}(\mathrm{z}) 12$ & FBst0031191 & Negative \\
\hline 33402 & $\mathrm{Su}(\mathrm{z}) 12$ & FBst0033402 & Negative \\
\hline 31342 & Top2 & FBst0031342 & Negative \\
\hline \multicolumn{4}{|c|}{ overexpression lines } \\
\hline 15026 & Cap-D3 & | FBst0015026 & Negative \\
\hline 17627 & Cap-H2 & FBst0017627 & Negative \\
\hline \multicolumn{4}{|l|}{ alleles } \\
\hline 200 & $\mathrm{z}[1] / \mathrm{z}[\mathrm{a}]$ & FBst0000200 & Negative \\
\hline 1728 & $\mathrm{Pc}[1]$ & FBst0001728 & Negative \\
\hline 4247 & $\mathrm{Su}(\mathrm{Hw})[2] / \mathrm{Su}(\mathrm{Hw})[\mathrm{e} 04061]$ & FBst0004247 & Negative \\
\hline 1053 & $\mathrm{Su}(\mathrm{Hw})[8]$ & FBst0001053 & Negative \\
\hline 18224 & $\mathrm{Su}(\mathrm{Hw})[2] / \mathrm{Su}(\mathrm{Hw})[\mathrm{e} 04061]$ & FBst0018224 & Negative \\
\hline 59959 & $\mathrm{Su}(\mathrm{Hw})[\mathrm{v}]$ & FBst0059959 & Negative \\
\hline 1059 & $z[1] / z[a]$ & FBst0001059 & Negative \\
\hline
\end{tabular}


Table S1. D. melanogaster stocks used to screen regulators of yellow spotted expression

\section{Materials and Methods}

Fly husbandry

Flies were raised on a standard cornmeal-agar medium and grown at $22^{\circ} \mathrm{C}$. All experiments were carried out at $25^{\circ} \mathrm{C}$, except for the RNAi screen which was carried out at $29^{\circ} \mathrm{C}$ unless stated differently.

Fly lines

\begin{tabular}{|c|c|}
\hline BDSC stock number & Genotype \\
\hline 24480 & $\mathrm{y}[1] \mathrm{M}\{3 \mathrm{xP3}-\mathrm{RFP} . \operatorname{attP}\} \mathrm{ZH}-2 \mathrm{~A} w\left[{ }^{*}\right] ; \mathrm{M}\{$ vas-int.Dm\}ZH-102D \\
\hline 32107 & $\mathrm{y}[1] \mathrm{w}[67 \mathrm{c} 23] \mathrm{P}\{\mathrm{y}[+\mathrm{t} 7.7]=$ CaryP $\}$ attP18 \\
\hline 24865 & $\mathrm{y}[1] \mathrm{M}\{$ vas-int.Dm $\} \mathrm{ZH}-2 \mathrm{~A} w[*] ;$ PBac $\{\mathrm{y}[+]-\operatorname{attP}-3 \mathrm{~B}\} \mathrm{VK} 00016$ \\
\hline 24871 & $\mathrm{y}[1] \mathrm{M}\{$ vas-int.Dm $\} \mathrm{ZH}-2 \mathrm{~A} w[*] ; \operatorname{PBac}\{\mathrm{y}[+]-\operatorname{attP}-3 \mathrm{~B}\} \mathrm{VK} 00033$ \\
\hline 851 & $\mathrm{y}[1] \mathrm{w}[67 \mathrm{c} 23] \mathrm{P}\{\mathrm{y}[+\mathrm{mDint} 2]=\mathrm{Crey}\} 1 \mathrm{~b} ; \mathrm{D}\left[{ }^{*}\right] / \mathrm{TM} 3, \mathrm{Sb}[1]$ \\
\hline- & $\mathrm{w}[*] ; \mathrm{wg}[\mathrm{Sp}-1] / \mathrm{CyO} ; \mathrm{MKRS} / \mathrm{TM} 6 \mathrm{~B}$ \\
\hline 33907 & $\left.\mathrm{y}[1] \mathrm{sc}{ }^{*}\right] \mathrm{v}[1] \operatorname{sev}[21] ; \mathrm{P}\{\mathrm{y}[+\mathrm{t} 7.7] \mathrm{v}[+\mathrm{t} 1.8]=$ TRiP.HMS00849 $\}$ attP2 \\
\hline 29734 & $\mathrm{y}[1] \mathrm{sc}\left[{ }^{*}\right] \mathrm{v}[1] \mathrm{sev}[21] ; \mathrm{P}\{\mathrm{y}[+\mathrm{t} 7.7] \mathrm{v}[+\mathrm{t} 1.8]=$ TRiP.HM05202 $\}$ attP2 \\
\hline 31191 & $\mathrm{y}[1] \mathrm{v}[1] ; \mathrm{P}\{\mathrm{y}[+\mathrm{t} 7.7] \mathrm{v}[+\mathrm{t} 1.8]=$ TRiP.JF01706 $\}$ attP2 \\
\hline 31342 & $\mathrm{y}[1] \mathrm{v}[1] ; \mathrm{P}\{\mathrm{y}[+\mathrm{t} 7.7] \mathrm{v}[+\mathrm{t} 1.8]=\mathrm{TR}$ iP.JF01300 $\}$ attP2 \\
\hline 31612 & $\mathrm{y}[1] \mathrm{v}[1] ; \mathrm{P}\{\mathrm{y}[+\mathrm{t} 7.7] \mathrm{v}[+\mathrm{t} 1.8]=$ TRiP.JF01396 $\}$ attP2 \\
\hline 33903 & $\mathrm{y}[1] \mathrm{sc}\left[{ }^{*}\right] \mathrm{v}[1] \operatorname{sev}[21] ; \mathrm{P}\{\mathrm{y}[+\mathrm{t} 7.7] \mathrm{v}[+\mathrm{t} 1.8]=\mathrm{TRiP} . \mathrm{HMS} 00845\}$ attP2 \\
\hline 32473 & $\mathrm{y}[1] \mathrm{sc}\left[{ }^{*}\right] \mathrm{v}[1] \mathrm{sev}[21] ; \mathrm{P}\{\mathrm{y}[+\mathrm{t} 7.7] \mathrm{v}[+\mathrm{t} 1.8]=\mathrm{TRiP} . \mathrm{HMS} 00473\}$ attP2/TM3, Sb[1] \\
\hline 32995 & $\mathrm{y}[1] \mathrm{sc}[*] \mathrm{v}[1] \operatorname{sev}[21] ; \mathrm{P}\{\mathrm{y}[+\mathrm{t} 7.7] \mathrm{v}[+\mathrm{t} 1.8]=\mathrm{TRiP} . \mathrm{HMS} 00795\}$ attP2 \\
\hline 33402 & $\mathrm{y}[1] \mathrm{sc}\left[{ }^{*}\right] \mathrm{v}[1] \mathrm{sev}[21] ; \mathrm{P}\{\mathrm{y}[+\mathrm{t} 7.7] \mathrm{v}[+\mathrm{t} 1.8]=\mathrm{TRiP} . \mathrm{HMS} 00280\}$ attP2/TM3, Sb[1] \\
\hline 42536 & $\mathrm{y}[1] \mathrm{v}[1] ; \mathrm{P}\{\mathrm{y}[+\mathrm{t} 7.7] \mathrm{v}[+\mathrm{t} 1.8]=\mathrm{TRiP} . \mathrm{HMJ} 02105\}$ attP40 \\
\hline 33659 & $\mathrm{y}[1] \mathrm{sc}\left[{ }^{*}\right] \mathrm{v}[1] \mathrm{sev}[21] ; \mathrm{P}\{\mathrm{y}[+\mathrm{t} 7.7] \mathrm{v}[+\mathrm{t} 1.8]=\mathrm{TRiP} . \mathrm{HMS} 00066\}$ attP2 \\
\hline 33704 & $\mathrm{y}[1] \mathrm{sc}[*] \mathrm{v}[1] \mathrm{sev}[21] ; \mathrm{P}\{\mathrm{y}[+\mathrm{t} 7.7] \mathrm{v}[+\mathrm{t} 1.8]=\mathrm{TRiP} . \mathrm{HMS} 00581\}$ attP2 \\
\hline 34069 & $\mathrm{y}[1] \mathrm{sc}\left[{ }^{*}\right] \mathrm{v}[1] \mathrm{sev}[21] ; \mathrm{P}\{\mathrm{y}[+\mathrm{t} 7.7] \mathrm{v}[+\mathrm{t} 1.8]=\mathrm{TRiP} . \mathrm{HMS} 00051\}$ attP2 \\
\hline 33906 & $\mathrm{y}[1] \mathrm{sc}\left[{ }^{*}\right] \mathrm{v}[1] \operatorname{sev}[21] ; \mathrm{P}\{\mathrm{y}[+\mathrm{t} 7.7] \mathrm{v}[+\mathrm{t} 1.8]=\mathrm{TRiP} . \mathrm{HMS} 00848\}$ attP2 \\
\hline 33945 & $\mathrm{y}[1] \mathrm{sc}\left[{ }^{*}\right] \mathrm{v}[1] \operatorname{sev}[21] ; \mathrm{P}\{\mathrm{y}[+\mathrm{t} 7.7] \mathrm{v}[+\mathrm{t} 1.8]=\mathrm{TRiP} . \mathrm{HMS} 00896\}$ attP2 \\
\hline 33946 & $\mathrm{y}[1] \mathrm{sc}[*] \mathrm{v}[1] \operatorname{sev}[21] ; \mathrm{P}\{\mathrm{y}[+\mathrm{t} 7.7] \mathrm{v}[+\mathrm{t} 1.8]=\mathrm{TRiP} . \mathrm{HMS} 00897\}$ attP2/TM3, Sb[1] \\
\hline 61180 & $\mathrm{y}[1] \mathrm{sc}\left[{ }^{*}\right] \mathrm{v}[1] \operatorname{sev}[21] ; \mathrm{P}\{\mathrm{y}[+\mathrm{t} 7.7] \mathrm{v}[+\mathrm{t} 1.8]=\mathrm{TRiP} . \mathrm{HMC} 05150\}$ attP40 \\
\hline 34006 & $\mathrm{y}[1] \mathrm{sc}\left[{ }^{*}\right] \mathrm{v}[1] \operatorname{sev}[21] ; \mathrm{P}\{\mathrm{y}[+\mathrm{t} 7.7] \mathrm{v}[+\mathrm{t} 1.8]=\mathrm{TRiP} . \mathrm{HMS} 00970\}$ attP2 \\
\hline
\end{tabular}




\begin{tabular}{|c|c|}
\hline 35297 & $\mathrm{y}[1] \mathrm{sc}\left[{ }^{*}\right] \mathrm{v}[1] \operatorname{sev}[21] ; \mathrm{P}\{\mathrm{y}[+\mathrm{t} 7.7] \mathrm{v}[+\mathrm{t} 1.8]=$ TRiP.GL00199 $\}$ attP2 \\
\hline 61903 & $\mathrm{y}[1] \mathrm{v}[1] ; \mathrm{P}\{\mathrm{y}[+\mathrm{t} 7.7] \mathrm{v}[+\mathrm{t} 1.8]=\mathrm{TRiP} . \mathrm{HMJ} 23458\}$ attP40 \\
\hline 40850 & $\mathrm{y}[1] \mathrm{v}[1] ; \mathrm{P}\{\mathrm{y}[+\mathrm{t} 7.7] \mathrm{v}[+\mathrm{t} 1.8]=\mathrm{TRiP} . \mathrm{HMS} 02017\} \mathrm{attP} 40$ \\
\hline 42926 & $\mathrm{y}[1] \mathrm{sc}\left[{ }^{*}\right] \mathrm{v}[1] \operatorname{sev}[21] ; \mathrm{P}\{\mathrm{y}[+\mathrm{t} 7.7] \mathrm{v}[+\mathrm{t} 1.8]=\mathrm{TRiP} . \mathrm{HMS} 02619\} \mathrm{attP} 40$ \\
\hline 27993 & $\mathrm{y}[1] \mathrm{v}[1] ; \mathrm{P}\{\mathrm{y}[+\mathrm{t} 7.7] \mathrm{v}[+\mathrm{t} 1.8]=\mathrm{TRiP} . J F 02826\} \mathrm{attP} 2$ \\
\hline 28343 & $\mathrm{y}[1] \mathrm{v}[1] ; \mathrm{P}\{\mathrm{y}[+\mathrm{t} 7.7] \mathrm{v}[+\mathrm{t} 1.8]=\mathrm{TRiP} . J \mathrm{~J} 02979\} \mathrm{attP} 2$ \\
\hline 31941 & $\mathrm{y}[1] \mathrm{v}[1] ; \mathrm{P}\{\mathrm{y}[+\mathrm{t} 7.7] \mathrm{v}[+\mathrm{t} 1.8]=\mathrm{TR}$ iP.JF02232 $\}$ attP2 \\
\hline 200 & $\mathrm{z}[1] \mathrm{w}[11 \mathrm{E} 4]$ \\
\hline 1059 & $y[1] z[a] w[11 E 4]$ \\
\hline 1728 & Pc[1]/TM1 \\
\hline 51635 & $\mathrm{y}[1] \mathrm{w}[*] ; \mathrm{P}\left\{\mathrm{w}\left[+\mathrm{m}^{*}\right]=\mathrm{nSyb}-\mathrm{GAL} 4 . \mathrm{S}\right\} 3$ \\
\hline
\end{tabular}

\section{Constructs \& Transgenics}

Constructs injected in D. biarmipes:

UAS-shRNA-tra: The shmir against transformer was created using the protocol from (Haley, B. et al. 2008)(31). The two shmirs we designed are shtra4.1: 5'-GACAGACTCCTTTCGACATAA-3' and shtra4.2: 5'-GCAAAGGAGTCCTCATCGGTA-3'. The vector (pNE3) that contained both of the transgenes was subcloned to a piggyBac vector using the In-Fusion ${ }^{\circledR}$ HD Cloning Kit from Takara with the primers pNE3_UAS_to_pBac.F: 5'-TACGCGTACGGCGCGCCGCTTCTGCAT CTCTCCGGATCCAAGC-3' and pNE3_UAS_to_pBac.R: 5'-GTCGACCTAGGCGCGCCGAT CCAGACATGATAAGATACATTGATG-3'.

UAS-Sxl: The SxI coding sequence was cloned from whole fly cDNA with the primers SxI.F: 5'GATCATGTACGGCAACAATAATCCG-3' and SxI.R: 5'-GATCTTATAAGTAAGGATAAT GGTACTTCCG-3' and then inserted using TOPO-TA into the pCR8 vector. It was then subcloned to a UAS-piggyBac vector with an LR reaction.

UAS-shRNA-mod(mdg4): The shmir against $\bmod (\operatorname{mdg} 4)$ we used is 5'TTCGTGTTGAAGTTGTTCCAG-3' and cloned in piggyBac Gateway vector ligating sh_mod(mdg4)top: 5'-CTAGCAGTCTGGAACAACATCAACACGTATAGTTATATTCAAG CATATTCGTGTTGAAGTTGTTCCAGGCC-3' and sh_mod(mdg4)bot: 5'-TCGAGGCCTGG AACAACTTCAACACGAATATGCTTGAATATAACTATACGTGTTGATGTTGTTCCAGACTG-3' between the restriction sites PspXI and Nhel. 
$y^{10 k b}$ : This construct was cloned at the Ascl site of a piggyBac vector using the In-Fusion ${ }^{\circledR} \mathrm{HD}$ Cloning Kit. The primers used for PCR on D. biarmipes genomic DNA were 1F: 5'TACGCGTACGGCGCGCCATCGATAATCGCCC GATTACCG-3', CTTCTATTGGGTTCTTTCTTAGCCGGAAAT-3', 2F: 5'- AGAACC CAATAGAAGTTCCAGAAAAGTGAC-3' and 2R: 5'- GTCGACCTAGGCGCGAGCATACT TACAGATACTCCTCATTTTCTATTTATGATG-3'.

These plasmids were injected in D. biarmipes embryos at $100.0 \mathrm{ng} / \mu \mathrm{l}$ along with the helper plasmid (also at $100.0 \mathrm{ng} / \mu \mathrm{l}$ ).

Constructs injected in D. melanogaster:

All the constructs have been established using the In-Fusion ${ }^{\circledR} \mathrm{HD}$ Cloning Kit.

$y^{25 k b}$ : This construct was cloned in the piggyBac vector at the Ascl site using the In-Fusion ${ }^{\circledR} \mathrm{HD}$ Cloning Kit and the following set of primers: 3F: 5'-TACGCGTACGGCGCGCCGAGGATTCT GCCAGATCCCGG-3', 3R: 5'-ATTATCGATGGC GCGAAACAATCGCAGCGATCTCCC CA-3', 1F, 1R, 2F, 2R， 4F: 5'-GATgAtAggAtATtT TAAATCACGAgGAAACGAATC TtAAACACGgG-3'， 4R: 5'TAAATAAACTTAATTTAA ATAAAAAAGCCCTTTTCCCGG-3', 5F: $\quad$ 5'-AATTAAGTTT

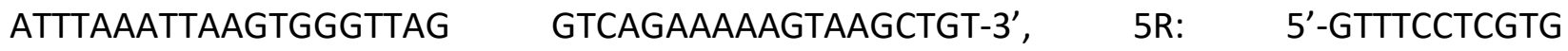
ATTTCACGCTGCCGGTGGG-3'.

$y:: m C^{2}$ erry ${ }^{25 k b}$ : The $m$ Cherry with the Waldo linker was cloned from the $p J E T-m C h e r r y$ vector using the primers: mCh1F: 5'- CCAGGGTTCCGCTGGCTCCGC-3' and mCh1R: 5'-GGGTTGGGTTAC TTGTACAGCTCGTCCATGCCGC-3'. This was inserted in the $y^{25 k b}$ vector to create the $y:: m C h e r r y^{25 k b}$ transgene using the Stul sites along with two fragments from the vector cloned with the primers: 6F: 5' - TTGAGGTGCCCAAGGCCTACATCTTCA-3', 6R: 5' - CCAGCGGAACCCTGGTGCTGGTGG-3', 7F: 5'- CAAGTAACCCAACCCGTGCACGG-3' and 7R: 5'- ATCTTAATCTTAAGGCCTCGTCTTTGGAG-3'. The $y:: m C^{2}{ }^{2} r y^{25 b}$ was then subcloned into the pWalium20 vector using the sites Aatll and Notl and the primers 8F: 5'- TCGAATGGCCATGGGACGTCTTTCCATAGGCTCCGCCCC-3' and 8R: 5'TCTAGAGTCGCGGCCGCCGAATTGATCCGGAGAGC-3'.

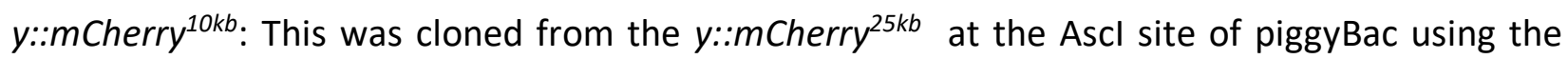
primers: 8F: 5' 5 $^{\prime}$ TACGCGTACGGCGCGCCATCGATAATCGCCCGATTACCG-3' and 8R: GTCGACCTAGGCGCGAGCATACTTACAGATACTCCTCATTTTCTATTTATGATG-3' and then subcloned to pWalium20 using the same strategy as for the $y^{25 k b}$. 
$y:: m$ Cherry ${ }^{10 k b \Delta i n t r o n}:$ This was cloned from the $y:: m$ Cherry $^{10 k b}$ at the Ascl site of piggyBac using the primers: 8F, 8.1R: 5'- AGGGATGCCATCTCGCCAGCGGG-3', 8.2F: 5'- CGAGATGGCAT CCCTGCCACTCT-3' and 8R. Then it was subcloned to a chimeric version of pWalium20 and piggyBac using the same strategy as previously described.

The plasmids were injected in the following stocks (BDSC stock numbers) : \#24480 or \#32107 (chromosome X), \#24865 (chromosome II), \#24871 (chromosome III). Following the injection in \#24480, we used the line \#851 to remove the 3xP3-RFP (flanked by two loxP sites) marking the attP site. Plasmid were injected at 100.0 ng/ul. For injection in \#32107, plasmid was injected along with a helper plasmid encoding for $\phi C 31$ integrase $(100.0 \mathrm{ng} / \mathrm{ul})$.

\section{Generation of a y mutant in D. biarmipes with Crispr/Cas9}

The yellow mutant in D. biarmipes was created according to (Bassett \& Liu 2014)(32). The sgRNA used was 5'-CCCCAGAACGGCCTTCCCG-3', identified using the Target Finder of flyCRISPR (https://flycrispr.org/). The yellow mutant was screened based on the phenotype and confirmed by Sanger sequencing.

\section{Antibody staining}

We performed antibody staining using the Yellow antibody and the protocol from (Hinaux, H. et al. 2018)(33).

\section{Imaging}

Adult wings of five-days-old flies were mounted on Hoyer's medium according to (Arnoult, L. et al. 2013)(11) and imaged on a Leica Wild M420 Makroscop equipped with a ProgRes C5 ccd camera (Jenoptik, Germany).

Reporter expression and fluorescent antibody-staining were imaged on an MSV269 stereoscope with a DFC365 FX camera (Leica). Freshly hatched adults (<30 minutes) were collected and fixed in 5\% Formaldehyde/1X PBS. One wing per individual was dissected and mounted in Vectashield medium. 
Image registration prior to quantification was performed by setting manually nine points described in Fig. 2e using a reference wing and the Landmark Correspondences plugin of Fiji (method: least squares, alpha: 1.00, mesh resolution: 32, class: similarity).

Quantification of fluorescent wings was performed measuring mean gray value using Fiji in the a, $b$, and $p$ regions defined in Fig. 2E. Quantification of fluorescent abdomens was performed similarly but measuring all the $A 4, A 5$ and $A 6$ tergites.

For each experiment, all pictures were taken under the same settings. All images were uniformly enhanced using Adobe Photoshop.

\section{$\underline{\text { Statistics and plots }}$}

No statistical methods were used to predetermine sample size. The experiments were not randomized and investigators were not blinded to allocation during experiments and outcome assessment. Plots were created using RStudio (v. 1.2.1335). Each point represents an individual wing (one per individual) or abdomen. Data were analyzed using a Generalized Linear Model (GLM) with a Gamma distribution. When the GLM showed a statistically significant difference between groups, the test was followed by a multiple comparison (Tukey HSD) test with a Bonferroni correction method ( $R$ : glht function in multcomp package). 